\title{
Pipe breaks and estimating the impact of pressure control in water supply networks
}

\author{
Carlos Jara-Arriagada*, Ivan Stoianov \\ Department of Civil and Environmental Engineering, Imperial College London, SW7 2AZ \\ United Kingdom
}

\begin{abstract}
Deterioration and fracture of water supply pipes present a critical threat for the continuous provision of drinking water. The hydraulic pressure in pipes is recognised as an influential factor for the occurrence of pipe breaks. However, little evidence has been provided so far for the quantitative assessment of the impact of pressure control on reducing the number of pipe breaks. In this paper, we applied logistic regression with polynomial terms, and a sensitivity analysis to assess the potential impact of pressure control on reducing pipe breaks. A large data set of historic pipe breaks was used to develop and validate the presented method. Cast iron and asbestos cement pipes were examined in detail. Results showed that pipe breaks could be decreased by $18 \%$ to $30 \%$ by reducing the mean pressure in asbestos cement and cast iron pipes respectively. Pressure range reduction could potentially decrease more than half of pipe breaks for both pipe materials. These results showed the significant impact that managing the hydraulic pressure can provide to improve the reliability of water supply pipes.
\end{abstract}

Keywords: Pipe Breaks, Logistic Regression, Machine Learning, Pressure

Control, Water Supply Networks Management

\footnotetext{
* Corresponding author

Email addresses: cij18@imperial.ac.uk (Carlos Jara-Arriagada), ivan.stoianov@imperial.ac.uk (Ivan Stoianov)
}

Preprint submitted to Journal of Reliability Engineering \& System Safety January 19, 2021 


\section{Introduction}

The reliability of water supply infrastructure is critical for the continuous and uninterrupted provision of clean water. A major problem that affects the normal operation of water supply networks is the occurrence of pipe breaks.

${ }_{5}$ Current research suggests that pipe breaks result from complex interactions of physical, environmental and operational factors that impact the deterioration rate and breaks of pipes 1, 2, However, many causes for pipe breaks are still not fully understood and accounted for. For example, high levels or sudden changes of hydraulic pressure could be significant contributing factors for pipe breaks

10 3. 4. Nonetheless, there has been insufficient empirical evidence to develop methods that can quantitatively determine the impact of hydraulic pressure on pipe breaks in operational networks. Furthermore, it is unclear which metrics of the hydraulic pressure are most causative for the occurrence of pipe breaks; e.g. mean pressure, pressure range and pressure transients.

15 High hydraulic pressure leading to excess pressure in water supply networks can be controlled with the use of pressure reducing valves (PRVs), which commonly are membrane operated globe valves. PRVs dynamically reduce excess pressure in a network by introducing local energy losses. Recent developments in electronic pilots for PRVs allow for the implementation of different control profiles for PRVs that can either be time based (time modulation) or demand based (flow modulation) [5, 6. The control profiles can be remotely modified to facilitate different operational objectives. These technologies enable significant flexibility in managing the pressure in large scale water supply networks. The reduction in pressure must not result in pressure that is below the minimum regulatory pressure for serviceability; e.g. a surrogate value of $15 \mathrm{~m}$ pressure head (1.5 bar) in water distribution pipes is used in the UK for the minimum regulatory pressure.

Reliable estimation of the impact of hydraulic pressure control on pipe breaks would allow operators to quantify extra benefits of pressure management on so reducing the number of pipe breaks and increasing the life cycle of ageing in- 
frastructure. Currently, the benefits from implementing pressure management are evaluated only on the basis of potential leakage reduction, for which the relationships of pressure and leakage are relatively well understood 7, 6, 8. Pressure management schemes are only implemented if the benefits exceed the installation and maintenance costs [7. Although water utilities recognise that the reduction in hydraulic pressure may decrease pipe breaks [7, 4, 9, the lack of empirical evidence and robust estimation methods have limited the inclusion of the potential reduction in pipe breaks in cost-benefits models for pressure management schemes [7].

Current research has suggested causation of the occurrence of pipe breaks with high pressure and pressure variations in operational networks. Rezaei et al. 4. investigated a case study of 48 DMAs and reported that pipe break rates had a positive correlation with high pressure variations. Martinez-Codina et al. 3. implemented an statistical analysis to assess differences in the pressure 45 cumulative distribution function of pipes conditioned to break against random pipes. Their analysis comprised the evaluation of various hydraulic pressure metrics. It was observed that the pressure range, which is the difference between maximum and minimum pressure, provided the biggest difference in the presented cumulative distribution functions. Martinez-Garcia et al. 10 performed a spatiotemporally based clustering analysis to assess the correlation of consistent high pressure with pipe failure rates. Results from this study showed that there was a strong correlation between high pressure and pipe breaks in areas with high failure rates. However, weak correlations were also observed in other areas, suggesting that the effects of pressure could be spatially dependant.

${ }_{55}$ Further research is, however, required to allow operators to assess the benefits of active pressure control for the reduction of pipe breaks in water distribution and transmission pipes.

While previous studies on analysing the hydraulic pressure as a factor for pipe breaks are limited 17, 4, 11, 3, 12, 10, a multitude of published studies have ${ }_{60}$ investigated general models for pipe failures prediction. These models can be broadly categorised in mechanistic and data driven (see [13, 14, 15] for reviews). 
Mechanistic approaches usually require expensive, in field, data collection to analyse the deterioration state and estimate fracture of critical elements in the networks [16. Data driven approaches make use of historical datasets and several covariates to estimate pipe breaks probabilities or breaks rates. This can later be used to assist in the allocation of resources for rehabilitation and replacement [1]. Data driven models can provide different outputs depending on how the algorithms are adapted. Support vector machines and neural networks have been applied to predict the rate of pipe breaks [17, 18, while evolutionary polynomial regressions have been applied to predict the number of pipes breaks [19]. Boosted decision trees [20] and logistic regression [21, 22] have been utilised to predict pipes breaks for individual pipes.

This paper presents a novel method that combines a pipe break prediction model with a sensitivity analysis to assess the impact of pressure control on pipe

75 breaks. In this way, we studied to what extent predicted pipe breaks may vary with hydraulic pressure, which can be proactively controlled. The implemented algorithm utilised logistic regression with polynomial terms. As a result, three simple but robust pipe breaks prediction models that account for non-linearities were developed. The models were fitted with data from a large dataset with over so 20 years of historic pipe breaks records (pipe repairs records) from a water supply network in the UK. The hydraulic pressure metrics used in this study were the mean pressure and pressure range. The control (reduction) of these pressure metrics is done with the implementation of pressure management schemes and the installation of PRVs. Pressure transients have not been included in this 85 study as their analysis requires a long-term experimental data collection, which is beyond the scope of this study.

The structure of the paper is as follows: Section 2 describes the data preparation, and the implemented machine learning method and sensitivity analysis that investigates the impact of pressure control on pipe breaks. Section 3 90 presents an exploratory analysis of the case study, which was used for the data selection and model development. Section 4 describes the model prediction performance and the results of the sensitivity analysis. Section 5 discusses both 
the model prediction performance and sensitivity analysis outcomes. Finally, conclusions are drawn in Section 6.

\section{Methods}

We have applied logistic regression with polynomial terms (or features) to predict pipe breaks. After an assessment and validation of the algorithm for the considered case study, a sensitivity analysis was implemented to assess variations in predictions against changes in the utilised hydraulic pressure metrics.

\subsection{Data preparation}

We received historic records of pipe repairs for a period of approximately 25 years from a UK water company. The pipe repairs records are an approximation for pipe breaks; however, there is inherent uncertainty with the time of occurrence of pipe breaks. The dataset contained missing values or inconsistent information, which is common for operational data. These values were modified with nan or unknown labels. We did not remove records for the exploratory analysis. However, for the predictive analysis, only entries of unique pipes were used that contained all necessary information. Also, as the dataset contained different pipe materials, these were not mixed for modelling, since asbestos cement and cast iron, for example, have different structural properties 1 .

Before utilising the data in the models, log transformations were carried out for the variable length and the variable pressure range since these were rightskewed. We also implemented z-score normalisation, to normalise the range of the data for optimisation purposes.

\subsection{Logistic regression}

Logistic regression is a generalised linear model, which is commonly used for modelling data with binary dependant variables. It is a supervised learning algorithm for solving classification problems. Logistic regression was chosen for this study over alternative machine learning techniques for the following reasons: 
(1) It is easier to interpret even with the use of polynomials terms compared to other models such as neural networks or decisions trees. (2) The algorithm does not need to perform complex hyperparameter optimizations, which provides a more user-friendly and reproducible methodology for operators. (3) The use of polynomial terms expand the algorithm to capture non-linearities with a similar power to advanced machine learning methods. This is achieved, however, at the cost of being computationally expensive for large feature spaces.

The implementation of logistic regression for this study is as follows [23]:

Firstly, we compiled a dataset with $m$ pipe records from a water supply network. Each pipe record $i$ in the dataset contained a pair $\left(x^{(i)}, y^{(i)}\right)$, where: $x^{(i)}$ : was a vector of attributes, features or variables for the pipe $i$. This included, inter alia, the length of the pipe, diameter and year of installation.

$y^{(i)}$ : was the state of the pipe $i$ broken or no broken, as a binary outcome 1 or 0 .

It was desired an algorithm capable of using the variables $x$ of a pipe to provide the expected outcome $y$ of the pipe as broken or not. Therefore, it was sought a function that outputs results in the range 0 to 1 given variables $x$ that might be affected by parameters $\theta$. To accomplish this, the sigmoid function is commonly utilised as a hypothesis function $h_{\theta}$ :

$$
h_{\theta}=g\left(\theta^{T} x\right)=\frac{1}{1+e^{-\theta^{T} x}}
$$

where $g(z)=\frac{1}{1+e^{-z}}$ is called sigmoid function. In addition, we formulate:

$$
\begin{gathered}
p(y=1 \mid x ; \theta)=h_{\theta}(x) \\
p(y=0 \mid x ; \theta)=1-h_{\theta}(x)
\end{gathered}
$$

Eq.2 indicates that $h_{\theta}(x)$ is sought to represent the probability of the output $y$ being equal to 1 given a vector of variables $x$ parametrised by a vector of parameters $\theta$. Eq. 3 specifies that the probability of $y=0$ should be equal to 1 
minus the result of $h_{\theta}(x)$. Both expressions can then be condensed in one single expression called the Bernoulli distribution:

$$
p(y \mid x ; \theta)=h_{\theta}(x)^{y}\left[1-h_{\theta}(x)\right]^{1-y}
$$

Secondly, for our data set of $m$ pipes, it can be assumed that every observation was generated independently and identically distributed. This allows the use of the method of maximum likelihood estimation to determine the value of the parameters that maximise the probability of the data. For example, find the parameters for Eq. 4 that have the highest likelihood of fitting the observed data.

The likelihood function associated with the above distribution is:

$$
\begin{aligned}
L(\theta)=p(\vec{y} \mid X ; \theta) & =\prod_{i=1}^{m} p\left(y^{(i)} \mid x^{(i)} ; \theta\right) \\
& =\prod_{i=1}^{m} h_{\theta}\left(x^{(i)}\right)^{y^{(i)}}\left[1-h_{\theta}\left(x^{(i)}\right)\right]^{1-y^{(i)}}
\end{aligned}
$$

Furthermore, the expression of the log-likelihood can be determined as:

$$
\begin{aligned}
\ell(\theta) & =\log L(\theta) \\
& =\sum_{i=1}^{m} y^{(i)} \log h_{\theta}\left(x^{(i)}\right)+\left(1+y^{(i)}\right) \log \left[1-h_{\theta}\left(x^{(i)}\right)\right]
\end{aligned}
$$

Thirdly, the value of the parameters that maximise the above expression can be determined using nonlinear programming solvers. With the obtained parameters, the probability of break for every record is given by using Eq. 1. A threshold in the range 0 to 1 can be set for the given probability to differentiate breaks from no breaks.

\subsection{Model fitting}

160

\subsubsection{Feature selection}

A vector of features $x$ is required for every record in order to fit the logistic regression model. The features are obtained by the attributes given in the 
dataset. However, if the features are utilised as they are without interactions or polynomial terms, valuable information could be lost. For example, it is known that age does not correlate linearly with the occurrence of pipe breaks [24. Three stages occur in the life of pipelines [25, 26, 27]. In the first stage, a high number of breaks appears due to installation practices and material imperfections. The break rate is then reduced during the second stage. The third stage includes accelerated pipe deterioration and failures. This non-linear behaviour could be captured and modelled with logistic regression by adding polynomial terms.

The addition of extra terms must be done systematically. Adding all computationally possible polynomial terms could result in overfitting for the model and may also add irrelevant variables, which may cause statistical problems such 175 as multicollinearity. The selection of the interactions and higher order terms to include could be manually performed, based on engineering knowledge. However, this would require several manual iterations to obtain the best model given the data. Instead, the following procedure was implemented: (1). the maximum polynomial order that avoids overfitting was explored by iteratively increasing the order of the features and comparing the results of training and k-fold crossvalidation; (2). a common feature selection algorithm was used to reduce the possible excess of features of the resulting model; and, (3). the behaviour of the variables was explored by a sensitivity analysis. If every augmented feature behaves in accordance with the available engineering knowledge, the model is accepted. For example, a decrease in pipe diameter increases break probability. Otherwise, the order of the feature is changed or the feature is removed, and the model fitted again.

The stepwise regression algorithm 28] was selected for the feature reduction analysis. This is a commonly used algorithm [29, 30, 31, that iterates from a base model and adds or removes terms given their impact on the model's performance to a selected criterion. Two common criteria used are the Akaike Information Criterion (AIC) and the Bayesian Information Criterion (BIC). The AIC aims to find the best model that approximates to the true model, (assumed not to 
be within the candidates set), and may tend to find complex models [32]. The

within a a two by two array. These are true positive (TP), true negative (TN) false positive (FP) and false negative (FN). Three evaluation measures from the confusion matrix are of interest to develop the ROC and PR curves:

$$
\begin{aligned}
& T P R=\frac{T P}{(T P+F N)} \\
& F P R=\frac{F P}{(T N+F P)}
\end{aligned}
$$




$$
P R E C=\frac{T P}{(T P+F P)}
$$

Where $T P R=$ True Positive Rate or Recall, $F P R=$ False Positive Rate, value for the threshold that maximises the F1-score generated by the model. F1-score is a measure of the results performance of a model when there is an imbalance in the dataset. The F1 score is defined as follows:

$$
F 1=\frac{2 T P}{(2 T P+F P+F N)}
$$

\subsubsection{K-fold cross-validation}

To ensure that the results of the fitted model can be generalised, a crossvalidation technique should be implemented. For this study k-fold cross-validation was chosen, whose formulation is as follows. The data $\mathrm{S}$ is randomly split into k disjoint subsets of $m / k$, where $m$ is the number of records in $S$, and $k$ is the 
number of desired subsets. In our case, we have chosen $k=5$. Then, the model

is evaluated as follows [36]:

for $j=1, \ldots, k$

the model is trained on $S_{1} \cup \ldots \cup S_{j-1} \cup S_{j+1} \cup \ldots \cup S_{k}$ to obtain the model $h_{j}$

Then, $h_{j}$ is tested on $S_{j}$, and their associated ROC and PR curves are calculated.

Generalised ROC and PR curves for the model are finally estimated as the average of the ROC and PR curves for all $h_{j}$.

\subsection{Sensitivity analysis}

The main objective of this study is to develop and validate a method to assess the impact of controlling particular pressure components (metrics) on reducing pipe breaks. Previous studies have indicated that pressure reduction could potentially provide a decrease in pipe breaks, but quantification of the real impacts has not been determined yet. For this study, a machine learning algorithm was applied to predict pipe breaks in a water supply network with the use of several covariates. After the proposed algorithm was validated for physical reliability and predictive capacity, then a procedure to assess how the number of breaks are affected by changes in pressure components was investigated. This procedure included a sensitivity analysis as follows:

The values of the mean pressure and pressure range in the network were varied by constant step reductions; i.e. the mean pressure obtained from the hydraulic model was iteratively reduced by steps of $5 \mathrm{~m}$ pressure head, and pipe breaks probabilities were recalculated for every step and stored for comparison. The results of the sensitivity analysis were then visualised in box-plots showing the change in the pipe breaks probabilities on the model results. Two points need to be noted. Firstly, if a threshold of $15 \mathrm{~m}$ pressure head was reached in the case of reducing the mean pressure, we stopped reducing the pressure in that pipe. Secondly, a minimum value of $0.01 \mathrm{~m}$ was set, which is similar to the mean pressure, in the case of the pressure range. 


\section{Case Study}

275 3.1. Dataset summary

This study was built on data provided by the water utility of a medium-sized city in the UK. One dataset contained descriptive characteristics of the pipes in the network. This included, id, length, diameter, installation year, material and classification of the surrounding soil in terms of ferrous corrosivity and fracture potential. Another dataset contained recorded pipe repairs, which occurred in the network, and, in some cases, the type of pipe break was also provided. The period of the pipe breaks in the dataset encompasses from December 1994 to early June 2019. The development of statistics in the exploratory analysis was carried out with pipe breaks between 1995 and 2018. All available information 285 was used for the modelling stage.

Maximum, minimum and mean pressure values for every node in the network were estimated from a hydraulic model. Average daily pressure values were derived for every pipe and associated in the dataset. General descriptive characteristics of the data are summarised in Table 1.

According to the dataset, three materials are predominantly used for the pipes installed in this water supply network; cast iron, plastic and asbestos cement. However, the plastic pipes, although being the second largest group, have considerably fewer records of breaks than cast iron and asbestos cement pipes (Table 1). This may be related to the young age of these pipes that does not extend beyond 40 years. Based on the previous antecedents, the analysis was focused on cast iron and asbestos cement pipes.

\subsection{Exploratory analysis}

A section of the water supply network, which was used in this study, with the various pipe materials is shown in Fig. 1. The central area has the highest density of pipes with a considerable number of cast iron pipes. Asbestos cement and polyethene pipes have been used as replacements for old cast iron pipes, and these can be found in specific sub-areas of the network. The oldest pipes in 


\begin{tabular}{ll}
\hline Description & Value \\
\hline Network length & $\sim 6,800 \mathrm{~km}$ \\
Main materials (\% of network length) & \\
$\quad$ Cast Iron & $45 \%$ \\
$\quad$ Polyethene & $22.3 \%$ \\
$\quad$ Asbestos Cement & $19 \%$ \\
Diameters (\% of network length) & \\
$\quad<=100$ & $43.7 \%$ \\
$\quad(100-150]$ & $29.9 \%$ \\
$\quad(150-250]$ & $14.0 \%$ \\
$\quad>250$ & $12.4 \%$ \\
Burst data period for exploratory analysis & $1995-2018$ \\
Number of breaks & 18,767 \\
Number of pipes affected by breaks & 12,396 \\
Percentage of the pipes affected by breaks per material & \\
Cast Iron & $69.3 \%$ \\
Polyethene & $4.6 \%$ \\
Asbestos Cement & $14.9 \%$ \\
\hline
\end{tabular}

Table 1: Case study 
the network correspond to cast iron pipes with installation dates between 1840 and 1960 .

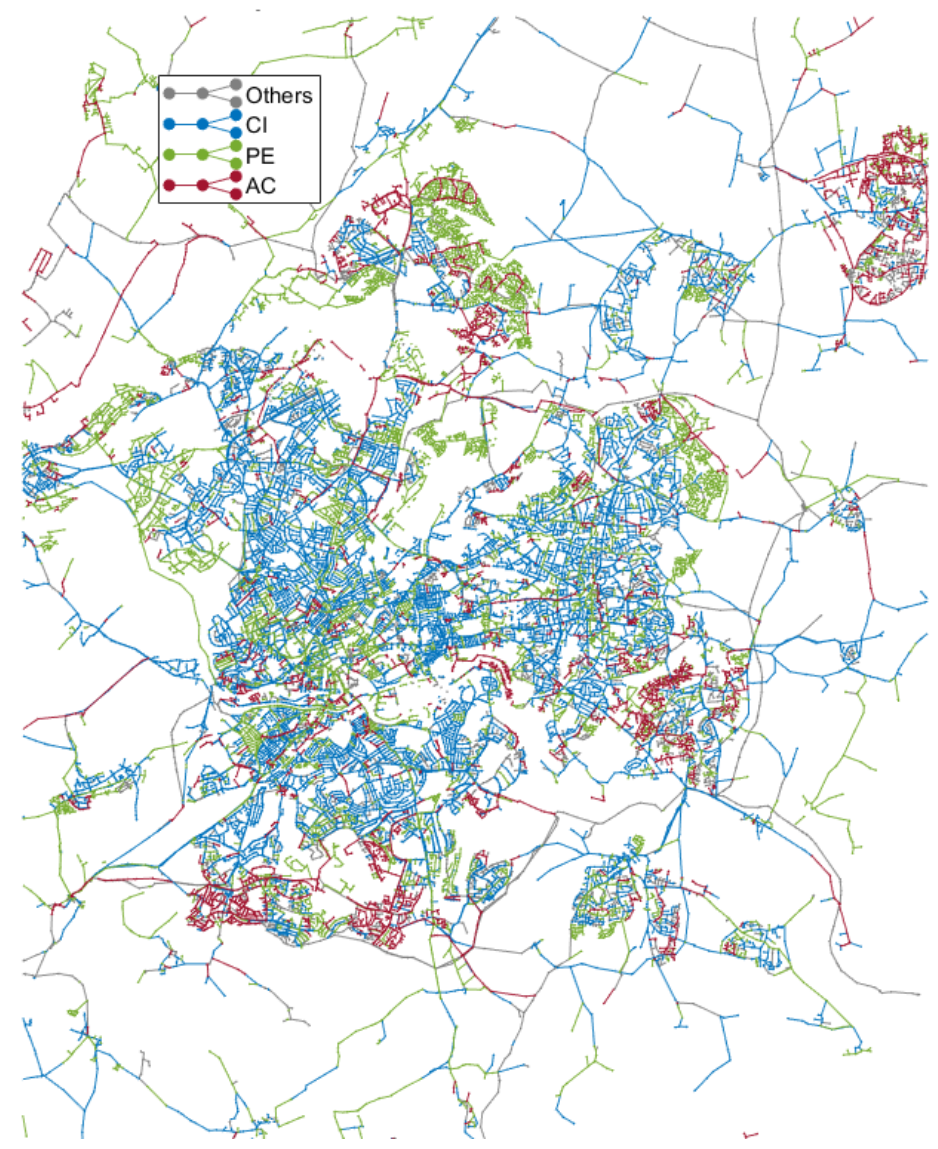

Figure 1: Central zone of the water distribution network is used for engineering design and strategic planning. No extended period simulation data, or actually measured pressure data, were used for this study. As a result, the pressure values are approximations for the mean, maximum and minimum pressures, which the network could experience over a period of time. Fig. 2 (a) shows the distribution of the mean pressure for the cast iron pipes. The bars in the plots are divided by colours that represent the proportion of the 
diameter range for each pressure band. Similarly, Fig. 2(b) shows the distribution of the pressure range for the cast iron pipes. The distribution of the mean pressure over the total pipe length can be considered symmetric with a peak of around $25 \mathrm{~m}$ to $35 \mathrm{~m}$ pressure head. The spread of the mean pressure ranges from around $25 \mathrm{~m}$ pressure head to few cases with over $75 \mathrm{~m}$ pressure head. The distribution of the pressure range is right skewed. There is little variability in the pressure range. The majority of the pipes are in the range of $0 \mathrm{~m}$ to 10 $\mathrm{m}$ pressure range with a high number of pipes in the range of $0 \mathrm{~m}$ to $2.5 \mathrm{~m}$. As stated, this pressure range is estimated from the hydraulic model and does not include the actual dynamic hydraulic conditions

In the analysed pipe breaks datasets, the attribute length represents the length of a pipe segment (id) based on arbitrary considerations relevant for building a hydraulic model from GIS. Therefore, the assigned length attribute has little relevance for the occurrence of a pipe break. However, it is an attribute of increasing exposition and probability to breaks (e.g. a larger number of pipe breaks occur in longer pipes). In Fig. 3 the $\mathrm{x}$-axis represents the resulting ranges of pipe lengths from grouping all pipes in the network in 10 groups with equal number of pipes (approximately 17,000 pipes in each bin). This analysis indicates that most pipe records are of pipes with short lengths and almost no breaks. Less than $5 \%$ of the total bursts have occurred in pipes with a length of up to $16.7 \mathrm{~m}$, and these pipes constitute around $60 \%$ of all pipes for the considered case study. Only pipes with length greater than $16.7 \mathrm{~m}$ have been used in the presented model. This approach reduces the number of excess zeros in the data without discarding pipes with break records.

The types of pipe breaks were provided for half of the records in the studied dataset. For the pipes with provided information about the break type, $30 \%$ described a circumferential break and the remaining 20\% included ferrule, hole, joint, and longitudinal breaks. The large fraction of circumferential breaks 340 suggested that pressure alone might not be the main cause of breaks in this network. Circumferential breaks may result from bending stresses produced by thermal contraction (low temperature in the pipe and surroundings), soil dif- 


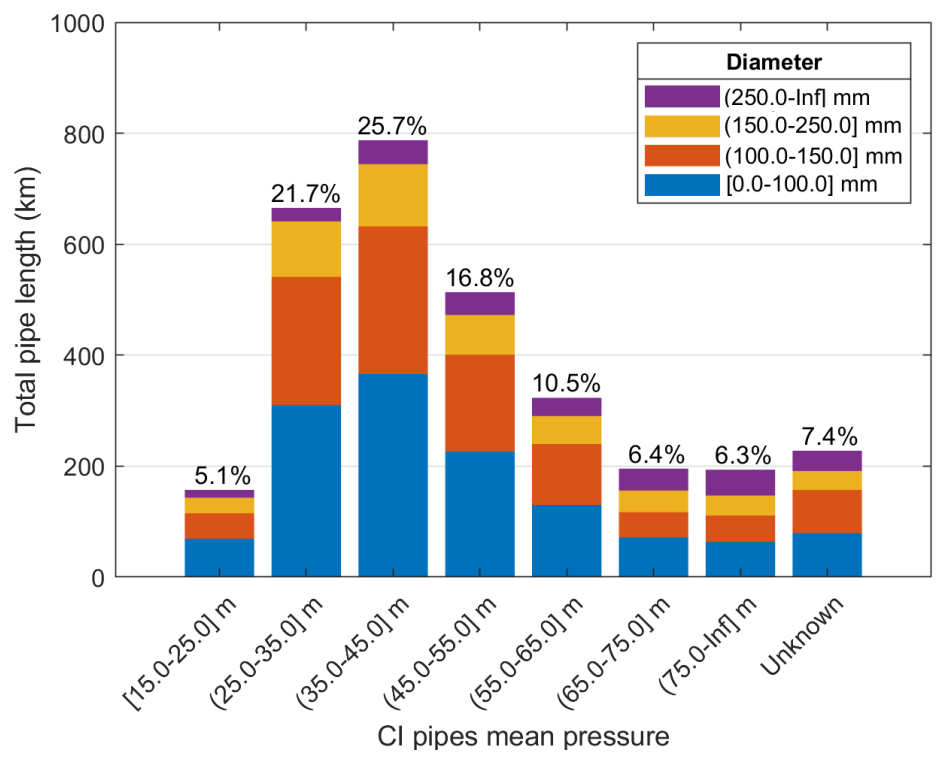

(a) Mean Pressure

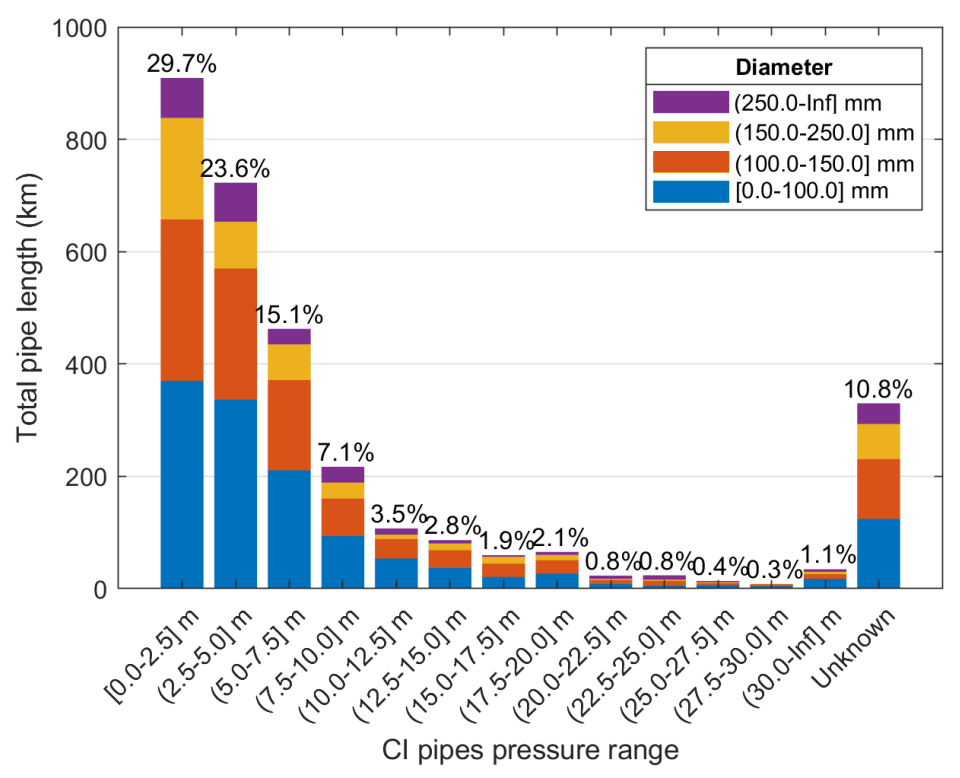

(b) Pressure Range

Figure 2: Distribution of pressure metrics for CI pipes 


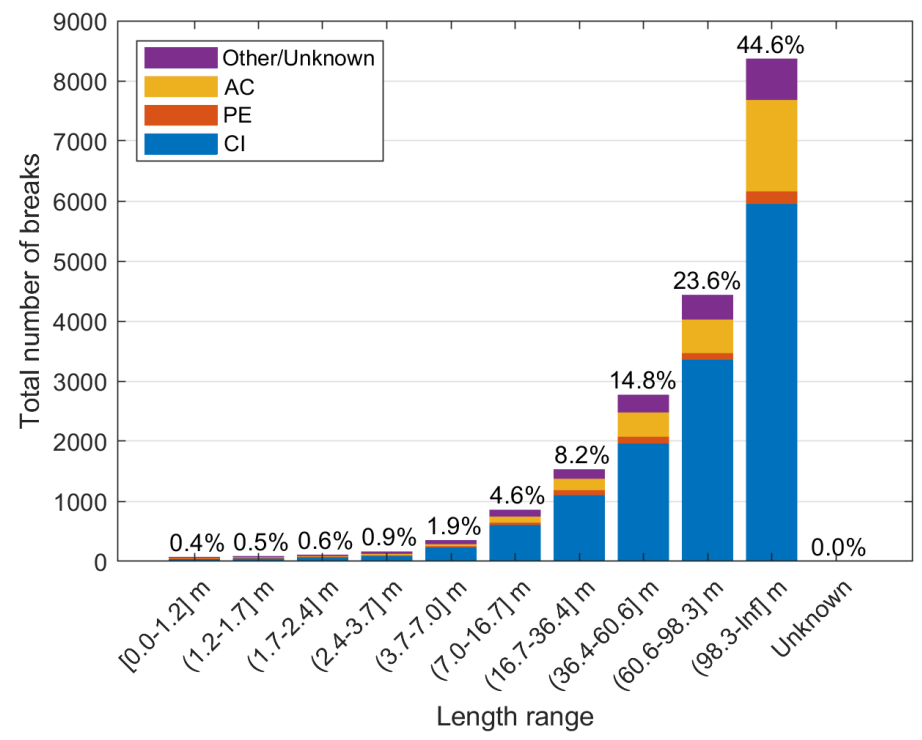

Figure 3: Number of breaks by pipe length range. The $\mathrm{x}$-axis represents the resulting length ranges from grouping all the pipes in the network in 10 groups with equal number of pipes (approximately 17,000 pipes in each bin). 
ferential movements, large cavities surrounding the pipe, inappropriate bedding practices or third parties. Longitudinal breaks, on the other hand, are associated to transverse stresses. These can be the result of the internal pressure in the pipe, and externals loads, such as cover loads, traffic, and soil expansion due to frosts [16].

In our dataset, the failures (pipe breaks) rate for cast iron pipes (Fig. 4 (a)) showed seasonality with the highest failure rate in winter months. The failure rate for asbestos cement pipes did not seem to be affected by seasonality (Fig. 4 (b)), but showed an increasing trend over time. Polythene pipes exhibited a low number of failures, which was not enough to provide a consistent failure rate trend, and thus their graph was omitted. The failure rate difference for cast iron pipes in winter-autumn and summer-spring seasons led to the creation of two cohorts, namely a winter-autumn and a summer-spring cast iron break cohorts.

\subsection{Cohorts for analysis and model building}

From the exploratory analysis, it was decided to implement three different pipe cohorts for modelling (Table 2): (1) asbestos cement cohort, including only asbestos cement pipes; (2) cast iron winter cohort, including only cast iron pipes with no breaks and breaks occurred in winter-autumn; and (3) cast iron summer cohort, which includes cast iron pipes with no breaks and cast iron pipes with breaks only in the summer-spring period. One break record per pipe was considered for modelling. In other words, if a pipe reported more than one break record the repeated break was not considered in the pipe cohort. Otherwise all 365 the characteristic of the pipe except age would not change and this would not provide extra information for modelling within this particular approach. Details of the variables used for every subset are presented in Table 3 .

The dataset provided information for soil fracture potential and soil ferrous corrosivity, derived from an external source. These variables were of interest in modelling. However, after a series of iterations, it was found that including these variables resulted in counterintuitive results, such as decreasing pipe breaks probabilities with higher values of ferrous corrosivity. This behaviour was also 


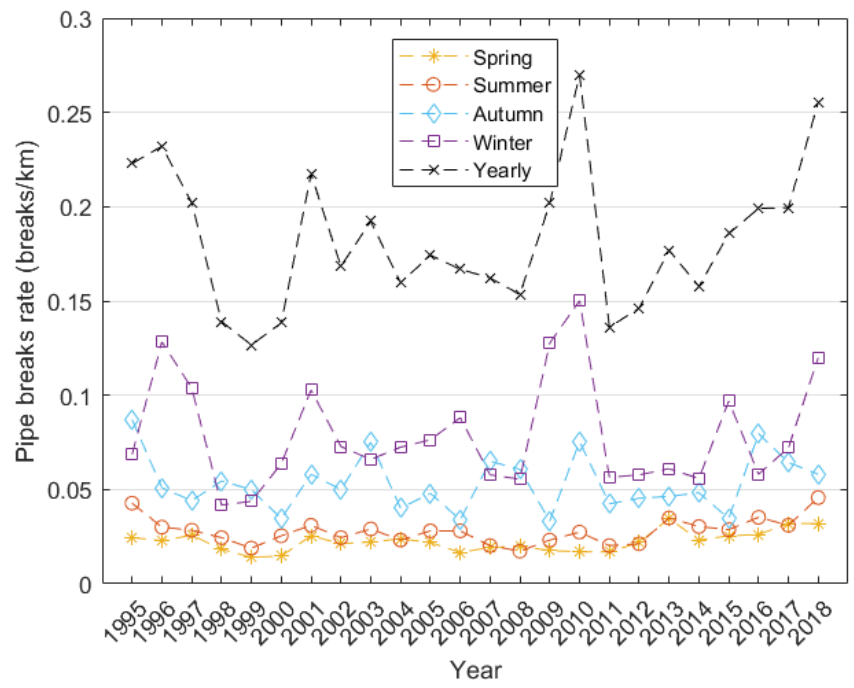

(a) Cast iron pipes

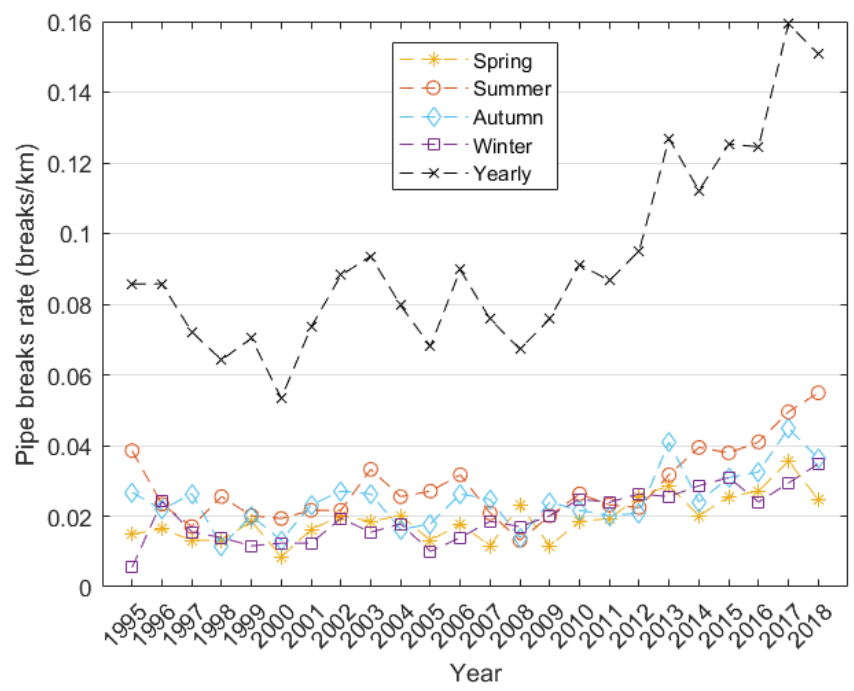

(b) Asbestos cement pipes

Figure 4: Seasonal break rates 


\begin{tabular}{llll}
\hline & Asbestos Cement & Cast Iron Winter & Cast Iron Summer \\
\hline Network length $(\mathrm{km})$ & $\sim 552$ & $\sim 1,736$ & $\sim 1,478$ \\
Number of records & 7,081 & 22,658 & 19,931 \\
Number of breaks & 947 & 4,731 & 2,004 \\
Break \% from the total & $13 \%$ & $21 \%$ & $10 \%$ \\
Ratio break no break & $1: 6.5$ & $1: 3.8$ & $1: 8.9$ \\
\hline
\end{tabular}

Table 2: Pipe cohorts for modelling

\begin{tabular}{llll}
\hline Variable & \multicolumn{3}{l}{ Range } \\
\hline & Asbestos Cement & Cast Iron Winter & Cast Iron Summer \\
\cline { 2 - 4 } Length (m) & $16.7-1,151$ & $16.7-1265$ & $16.7-1,265$ \\
Diameter (mm) & $50-250$ & $19-249$ & $19-249$ \\
Pressure Range (m) & $0.03-56.21$ & $0.03-94.7$ & $0.03-94.73$ \\
Mean Pressure (m) & $11.51-132.04$ & $8.99-133.3$ & $8.99-132.8$ \\
Customer Connections (n) & $0-94$ & $0-244$ & $0-244$ \\
Age (years) & $1-131$ & $23-191$ & $24-191$ \\
\hline
\end{tabular}

Table 3: Variables used as input for modelling

observed by [37. To avoid inconsistencies, it was decided to exclude these variables from the analysis.

\section{Results and Discussion}

This section presents and discusses results in two separate subsections. First, main results of the modelling approach and discussion of the selected model for every pipe cohort are presented. In the second subsection, analysis of the effect of pressure using the selected models, also for every pipe cohort, is discussed.

4.1. Main model results

\subsubsection{Asbestos cement cohort}

Exploration of the optimal polynomial degree for analysis showed that up to a 3rd degree polynomial for all variables, both training and validation provide similar results. Higher polynomial degrees show an overfitting pattern (Fig. 5 
(a)). Training results for both AUC ROC and PR curves increase as the polynomial degree increase. However, cross-validation results stagnate after increasing the model complexity to 4th degree polynomial and even decrease after 5 th degree. This shows that the model stops generalising properly over 3rd degree polynomials by capturing noise in the data.

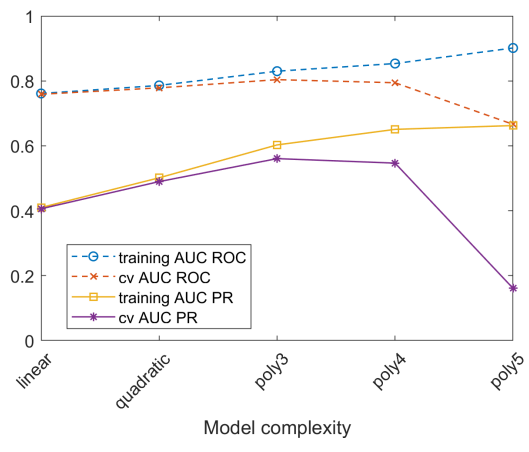

(a) AC cohort

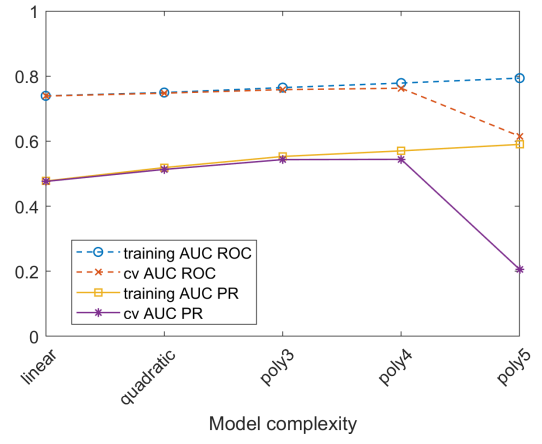

(b) CI winter cohort

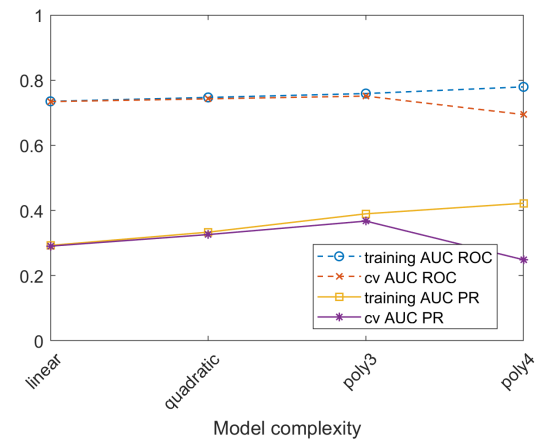

(c) CI summer cohort

Figure 5: Areas under curve (AUC) of the receiver operating characteristics (ROC) and precision-recall (PR) curves for training and cross-validation (cv) by increasing model complexity.

With the notion that up to a 3rd degree polynomial overfitting can be avoided, the stepwise regression algorithm was implemented to reduce the number of features. The original reduced model proposed alone by the stepwise regression included a cubic term of the feature age. However, this did not pro- 
vide realistic results. The model output probabilities of almost zero for all pipes when changing their ages over 80 years old. To avoid the previous issue, the degree of the feature age was allowed to be expanded only up to a quadratic term with the stepwise regression. This change provided better consistency (similar results to Fig. 61, however, the overall performance of the model decreased. The cross-validated AUC ROC decreased from 0.814 to 0.796 and the AUC PR decreased from of 0.581 to 0.526 (Table 4 .

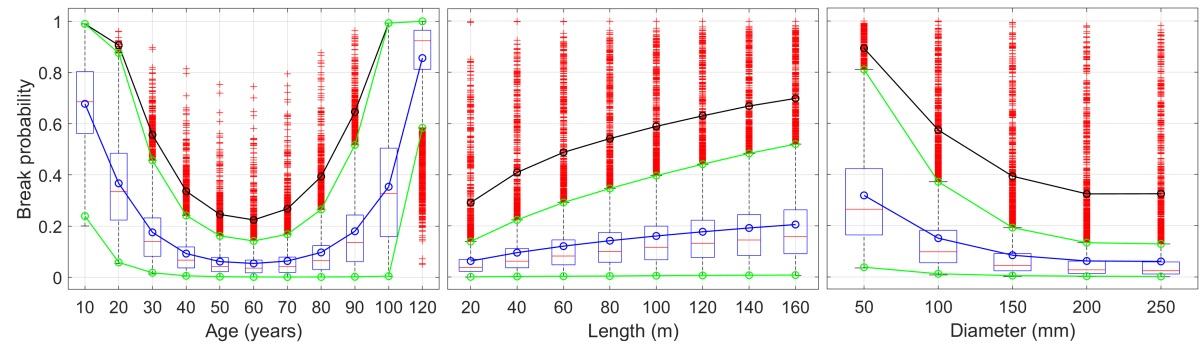

Figure 6: Coherence check for the asbestos cement model up to quadratic terms by varying values of age, length and diameter for all pipes. Similar trends were also obtained for both cast iron models.

The decrease in performance by limiting the age variable reached similar values to a quadratic model (Fig. 5 (a)). This prompted us to compare the difference in performance of the previous model and a stepwise regression model up to a quadratic degree. The comparison showed that the quadratic model performed slightly worse in prediction in almost all aspects except recall (Table 4. However, this model used 17 terms in comparison with the 48 terms used by the model that explored cubic terms (except for the age feature). Also the probability trends estimated with the model by varying age, length and diameter were consistent to engineering knowledge (Fig. 6). Despite the reduction in predictive power, it was decided that the simpler model up to quadratic terms would be more appropriate for inference and therefore selected for further analysis. Table 5 presents the resulting logistic regression models utilised for further analysis in every pipe cohort. 


\subsubsection{Cast iron winter cohort}

The approach, which was applied for the AC cohort, showed that for the CI cohort with winter breaks no overfitting was observed up to 4th degree polynomial (Fig. 5 (b)). However, the difference in performance between a quadratic and 4th degree model was less strong, and resulted in the selection of a model with interactions up to quadratic terms. The model outputs agreed with engineering knowledge.For example, the model estimates an increase in pipe breaks probabilities if the length of pipes was increased. In addition, the breaks probabilities increase if the diameter of pipes was decreased. An increase in both pressure metrics increases break probabilities. An increase in customer connections increases break probabilities. The age shows a consistent pattern with high break probability when pipes are recently installed and a decrease in break probability later in their operational life. However, the rise in break probability start to increase in general only after 140 years, which may be due to the quality of the records. The dataset contains pipes over 180 years old and many pipes over 100 years without any reported break that may influence the data fitting.

\begin{tabular}{llllll}
\hline Cohort & \multicolumn{3}{c}{ AC cohort } & CI winter cohort & CI summer cohort \\
\hline \multirow{2}{*}{ cv AUC ROC } & poly3 & poly3 $\left(\mathrm{age}^{2}\right)$ & quadratic & quadratic & quadratic \\
\cline { 2 - 6 } cv AUC PR & 0.814 & 0.796 & 0.78 & 0.748 & 0.744 \\
Threshold & 0.22 & 0.526 & 0.491 & 0.515 & 0.33 \\
cv F1-score & 0.53 & 0.501 & 0.24 & 0.26 & 0.17 \\
cv Recall & $53.7 \%$ & $42.3 \%$ & $48.8 \%$ & $54.7 \%$ & 0.36 \\
cv Precision & $49.5 \%$ & $57.6 \%$ & $46.4 \%$ & $42.9 \%$ & $43.6 \%$ \\
No features & 44 & 48 & 17 & 21 & $29.2 \%$ \\
coherence & no & yes & yes & yes & 19 \\
\hline
\end{tabular}

Table 4: Performance metrics by pipe cohorts and maximum polynomial degree explored by the stepwise regression using the Akaike Information Criterion (AIC). 


\begin{tabular}{ll}
\hline Cohort & Formula $\left[\operatorname{logit}(p)=\theta^{T} x\right]$ \\
\hline Asbestos cement & $\operatorname{logit}(p)=-2.77-0.92 \cdot A+0.33 \cdot A^{2}+0.52 \cdot L-0.7 \cdot D+$ \\
& $0.18 \cdot M P+0.24 \cdot C C+0.11 \cdot(M P \cdot A)+0.15 \cdot P R-0.05$ \\
& $C C^{2}+0.11 \cdot D^{2}-0.1 \cdot(D \cdot P R)-0.11 \cdot(D \cdot A)-0.07 \cdot(P R$ \\
& $C C)+0.07 \cdot(L \cdot C C)+0.06 \cdot(L \cdot P R)-0.05 \cdot(C C \cdot A)$ \\
& $\operatorname{logit}(p)=-1.89-0.68 \cdot A+0.29 \cdot A^{2}+0.43 \cdot L-0.46$ \\
& $D+0.31 \cdot C C+0.16 \cdot M P-0.08 \cdot(L \cdot C C)+0.06 \cdot L^{2}-$ \\
& $0.02 \cdot C C^{2}-0.04 \cdot M P^{2}-0.07 \cdot(L \cdot A)+0.06 \cdot(C C \cdot A)+$ \\
& $0.07 \cdot(D \cdot A)+0.05 \cdot(L \cdot D)+0.04 \cdot D^{2}-0.05 \cdot(D \cdot C C)+$ \\
& $0.03 \cdot(L \cdot M P)+0.04 \cdot(P R \cdot M P)-0.02 \cdot P R^{2}-0.01 \cdot P R$ \\
& $\operatorname{logit}(p)=-2.9-0.57 \cdot A+0.27 \cdot A^{2}+0.46 \cdot L-0.36$ \\
& $D+0.37 \cdot C C+0.09 \cdot L^{2}+0.17 \cdot M P-0.03 \cdot C C^{2}+0.09$. \\
& $(D \cdot A)+0.07 \cdot D^{2}-0.08 \cdot(L \cdot C C)+0.04 \cdot(C C \cdot A)-$ \\
& $0.05 \cdot(P R \cdot A)-0.03 \cdot\left(M P^{2}\right)+0.05 \cdot(P R \cdot M P)+0.05$. \\
& $(L \cdot P R)-0.05 \cdot(P R \cdot C C)+0.02 \cdot P R$ \\
\hline
\end{tabular}

Where: $L=$ length, $D=$ diameter, $A=$ age, $P R=$ pressure range, $M P=$ mean pressure, $C C=$ customer connections

Table 5: Covariates and coefficients for the logistic regression models utilised for analysis in every pipe cohort. 


\subsubsection{Cast iron summer cohort}

Similarly to the other two cohorts, a model with interactions and quadratic terms provided a similar performance to higher order degrees (Fig. 5 (c)). However, in this particular case, the precision of the model was considerable low. Although the model reached a cross-validation value of 0.744 for its AUC ROC which could be considered acceptable, it only achieved a value of 0.33 for its AUC PR. The value of the AUC PR curve is still far over the threshold that separates the model for a random classifier. For a threshold that optimises its F1-score, the model has a recall of $43.6 \%$ but a precision of $29.2 \%$. The meaning of this is as follows: Over all the pipes that are given to the model, this is capable of identifying almost half of the pipes that actually break within its predictions. However, from all the models' break predictions, only around $29 \%$ are true. Considering that breaks are a minority class within a large population, this still indicates predictive power of the model, but that can be further improved. When the predictive power of the model is analysed to assess no breaks predictions, the model has a recall of $87.2 \%$ and a precision of $93.5 \%$. This example can be extended to the other two cohorts.

Note that the results from this model need to be considered cautiously as these show higher signals of bias. The quality of the data and the features explored play an important role. More factors affecting pipe breaks in summer may be missing, which may have led to the low precision and to miss important interactions; for example, the inclusion of pressure transients.

\subsection{Sensitivity analysis}

The models obtained for the $\mathrm{AC}$ cohort and both CI cohorts were used to simulate the effects of pressure control. The original values provided from the hydraulic model for every pipe were decreased in steps until the regulatory requirement in England and Wales for minimum pressure head of $15 \mathrm{~m}$ in a water distribution pipe for the mean pressure. The pressure range was allowed to decrease for every pipe until almost 0 . The boxplots of Fig. 7 were developed to gain understanding of how the change in pressure affects the trends in the 
distribution of the predicted probabilities of the models. Tables 6 and 7 show the quantitative results of pressure reduction on the number of predicted breaks by the three models.

There is a reasonable number of outliers shown in the boxplots of Fig. 7

An explanation for this is the fact that most of the pipes used for modelling have not experienced pipe breaks (Table 22. Therefore, it is expected that most of the predictions given by the models will concentrate in low values. This lead to treat break predictions with high degree of confidence as outliers. The distance between the lower quartile and lower whisker represents $25 \%$ of the data with lowest break probability. It is expected that this would not have a high variation as pressure decreases since their break probability is already low. Aspects of interest are the distance between the upper whisker and upper quartile, and the spread of the outliers. Decrease of these ranges as pressure reduces indicates reduction of the break probability trend for the pipes with more chance to break. The thick black line indicates the mean position of the outliers while the blue line indicates the mean of the data. Depending of the optimal threshold selected to differentiate breaks from no breaks, slight changes may lead to a large reduction of predicted breaks. Consequently, Table 6 and Table 7 are provided to understand the actual quantitative changes in pipe break predictions for the models as pressure metrics decrease.

\subsubsection{Asbestos cement cohort}

Results of the sensitivity analysis for the asbestos cement cohort indicate that both mean pressure and pressure range have potential effects on reducing pipe breaks. There is a slight and constant decrease of the upper whisker for the boxplot that shows the predicted break probabilities as function of the mean pressure reduction (Fig. 7 (a)). This led to a decrease of 10\% in predicted breaks when applying a reduction of $10 \mathrm{~m}$ pressure head in every pipe. An 18\% reduction was observed by decreasing the mean pressure in every pipe up to 20 m (Table 6).

The reduction in pressure range was of particular interest as it suggests an 


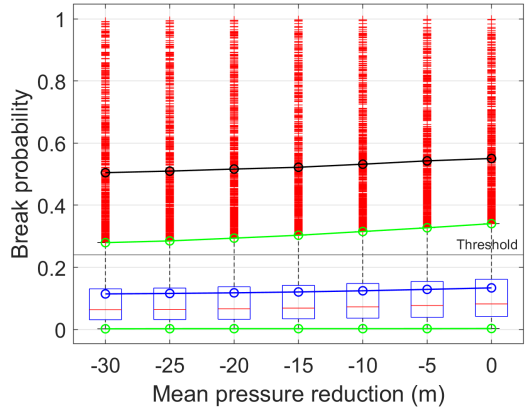

(a) AC cohort mean pressure

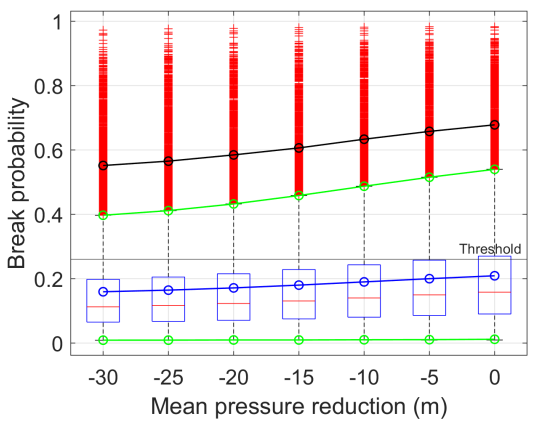

(c) CI cohort winter mean pressure

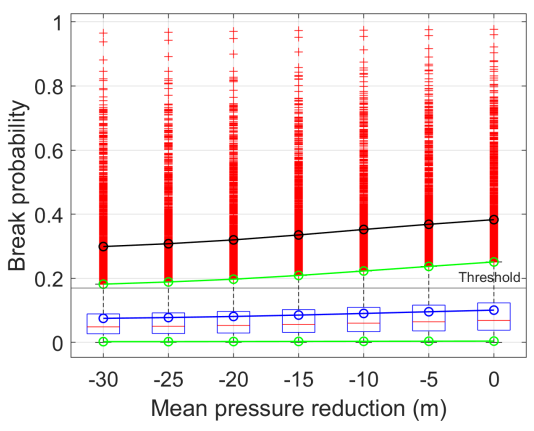

(e) CI cohort summer mean pressure

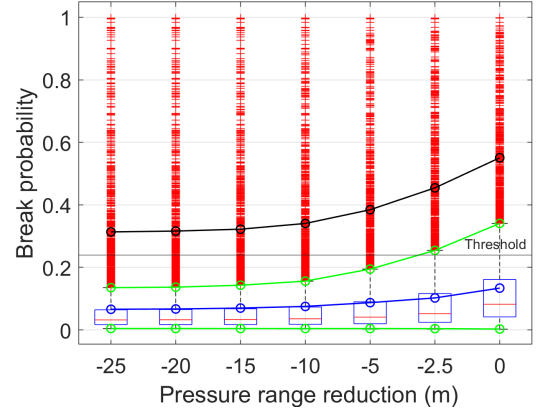

(b) AC cohort pressure Range

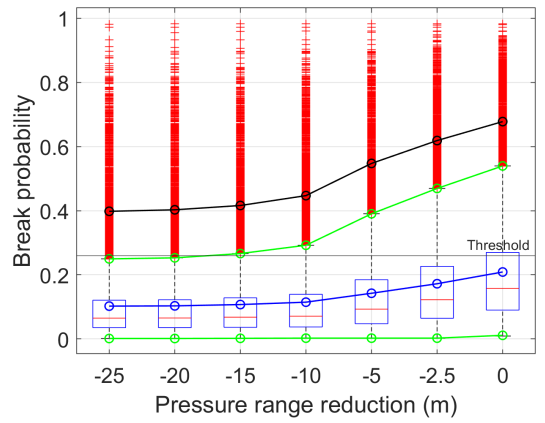

(d) CI cohort winter pressure range

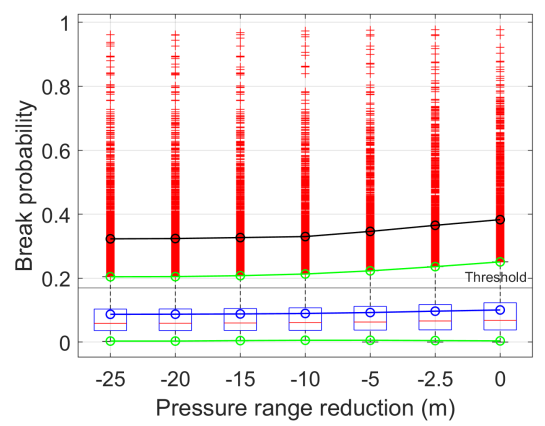

(f) CI cohort summer pressure range

Figure 7: Pipe breaks probabilities change by decreasing pressure metrics (from right to left). The $\mathrm{x}$-axis in the plots indicates the $\mathrm{mH}_{2} \mathrm{O}$ reduction applied to every pipe in the network if possible. 
almost exponential decrease in pipe breaks up to $10 \mathrm{~m}$ pressure range reduction (Fig. 7 (b)). After $10 \mathrm{~m}$ pressure range reduction the reduction in pipe break probabilities flattens. This may be a reflection of the quality of the data used. As shown in Fig. 2 the distribution of the pressure range is right skewed and with little variability for most of the pipes. A reduction in pressure range of $5 \mathrm{~m}$ pressure head leads to a decrease of $40 \%$ in pipe breaks as estimated by the model. A reduction in pressure range of $10 \mathrm{~m}$ pressure head leads to a high decrease of $54 \%$ in predicted pipe breaks (Table 7). These results indicate that reduction of pressure range may be an important factor to consider to reduce pipe breaks for asbestos cement pipes. These results however, should be confirmed with a highly calibrated model that truly reflect the pressure range distribution.

\subsubsection{Cast iron winter cohort}

The model for cast iron pipes winter cohort indicates an even higher potential for reducing pipe breaks with reducing both mean pressure and pressure range. A constant decrease in pipe break probabilities is estimated by the model when reducing the mean pressure for every pipe (Fig. 7 (c)) . This translates to a $16 \%$ reduction in the number of pipe breaks predicted by the model by a mean pressure decrease of $10 \mathrm{~m}$ for every pipe. The number of predicted breaks decrease by $30 \%$ with a mean pressure reduction of $20 \mathrm{~m}$. (Table 6).

The largest reduction in pipe breaks prediction is obtained by decreasing the pressure range. There is a steep decrease in the distance between the upper whisker and upper quartile as the pressure range reduces for every pipe. Also, the outliers tend to concentrate and approximate to the upper whisker (Fig. 7 (d)). Similar to the asbestos cement, the reduction in break probabilities flattens beyond a decrease in $10 \mathrm{~m}$ pressure range, possibly due to the pressure range distribution. High reductions in breaks predictions by $43 \%$ and $61 \%$ are given by the model when decreasing the pressure range in 5 and $10 \mathrm{~m}$ respectively.

Results from this model are of particular interest because of two reasons: (1) ${ }_{520}$ This model has the best predictive power from the three pipe cohorts analysed 
(Table 4), and (2) it also makes use of most of the available data (Table 2 ). This increases the confidence of the obtained results and the notion that pressure range may play an important role on the occurrence of pipes breaks. Another important consideration is that the model only considered cast iron pipes breaks occurred in winter. Therefore, it may be possible that high pressure range accentuates the effects of low temperature on cast iron pipes (e.g. the embrittlement of cast iron pipes at low temperatures combined with the impact of pressure components).

\subsubsection{Cast iron summer cohort}

Results of the sensitivity analysis indicate that mean pressure reduction also has positive impacts on this pipe cohort. There is a constant reduction of the upper whisker and the mean of the outliers as the mean pressure decrease (Fig.7] (e). A reduction of the mean pressure by $10 \mathrm{~m}$ and $20 \mathrm{~m}$ leads to $18 \%$ and $32 \%$ decrease in predicted breaks respectively (Table 4 .

Contrary to the CI winter cohort, the model for the CI summer cohort shows a lower impact of the pressure range on the probability of pipe breaks (Fig. 7 (f), Table 7p. Our hypothesis is that low temperature embrittles the cast iron material, and this exacerbates the impact of pressure range on pipe breaks in winter. The model for CI summer cohort is the most inaccurate model in terms of predictive power from the three models analysed. Therefore, results may be biased. The trends showed by this model, however, are consistent with the estimates from the other two models.

\section{Conclusions}

The ability to estimate the impact of pressure control on reducing pipe breaks and extending the life expectancy of ageing pipe infrastructure is particularly critical for water utilities because of financial constraints, regulatory changes and environmental issues. To address this challenge, we have outlined a novel approach that combines machine learning for the prediction of pipe breaks with 


\begin{tabular}{|c|c|c|c|c|}
\hline \multirow[t]{2}{*}{ Model } & \multirow[t]{2}{*}{$\mathrm{T}$} & \multicolumn{3}{|c|}{ Number of predicted pipe breaks ( $\%$ decrease) } \\
\hline & & Given pressure & $10 \mathrm{~m}$ reduction & $20 \mathrm{~m}$ reduction \\
\hline AC cohort & 0.24 & $1006(0 \%)$ & $910(10 \%)$ & $827(18 \%)$ \\
\hline CI winter cohort & 0.26 & $6023(0 \%)$ & $5078(16 \%)$ & $4215(30 \%)$ \\
\hline CI summer cohort & 0.17 & $2988(0 \%)$ & $2438(18 \%)$ & $2017(32 \%)$ \\
\hline
\end{tabular}

$\mathrm{T}$ indicates the threshold used for classification that maximises the $\mathrm{F} 1$ score for the models

Table 6: Effect of reducing the mean pressure in the model's predictions

\begin{tabular}{|c|c|c|c|c|}
\hline \multirow[t]{2}{*}{ Model } & \multirow[t]{2}{*}{$\mathrm{T}$} & \multicolumn{3}{|c|}{ Number of predicted pipe breaks (\% decrease) } \\
\hline & & Given pressure & $5 \mathrm{~m}$ reduction & $10 \mathrm{~m}$ reduction \\
\hline $\mathrm{AC}$ cohort & 0.24 & $1006(0 \%)$ & $603(40 \%)$ & $464(54 \%)$ \\
\hline CI winter cohort & 0.26 & $6023(0 \%)$ & $3417(43 \%)$ & $2348(61 \%)$ \\
\hline CI summer cohort & 0.17 & $2988(0 \%)$ & $2478(17 \%)$ & $2347(21 \%)$ \\
\hline
\end{tabular}

$\mathrm{T}$ indicates the threshold used for classification that maximises the F1 score for the models

Table 7: Effect of reducing the pressure range in the model's predictions 
a sensitivity analysis. This approach facilitates the quantification and understanding of how pipe breaks are affected as a function of the variation in two main pressure components; namely, mean pressure and pressure range. Other pressure components can also be included.

The proposed method was applied to a case study, and the results indicate a $18 \%$ decrease in the number of pipe breaks when reducing the mean pressure up to $20 \mathrm{~m}$ for asbestos cement pipes. For the same mean pressure reduction, $30 \%$ and $32 \%$ reduction in pipe breaks were estimated for cast iron pipes in winter and summer respectively. A reduction in $10 \mathrm{~m}$ pressure range suggests a major $54 \%$ reduction in predicted pipe breaks for asbestos cement pipes. Also, the same pressure range reduction suggests a major $61 \%$ reduction in pipe breaks for cast iron pipes in winter. This effect, however, was less pronounced for pipe breaks for cast iron pipes in summer months as the model suggests a reduction of $21 \%$ in pipe break predictions. The results, however, are influenced by the hydraulic pressure data, which was obtained from a hydraulic model. Actual pressure measurements with high spatial and temporal resolution, or a calibrated hydraulic model would be particularly beneficial to complement and expand the presented analysis.

This paper has described an approach to evaluate the effect of pressure management on pipe breaks. A large data set from a real water supply network was used. Next steps for future work should include the analysis of the effects of pressure transients, which were not included in this study. Another aspect that needs to be investigated is the temporal impact of pressure control for the suggested benefits to be realised. Finally, this study was based on observational data. To increase the confidence of the effects of pressure control on pipe breaks, an experimental study is encouraged. The findings of this paper together with the current related literature justify such study.

Overall, it has been presented through a systematic approach that reduction in mean pressure and pressure range have positive correlations on decreasing the number of pipe breaks. This indicates that pressure control has the potential to increase the reliability of water supply infrastructure and, in turn, the provi- 
analysts as an initial evaluation tool to assess the benefits of pressure control on pipe breaks. The quantitative results on reducing pressure for pipe breaks reduction can then be incorporated in cost benefit analysis.

\section{Acknowledgements}

\section{References}

[1] N. A. Barton, T. S. Farewell, S. H. Hallett, T. F. Acland, Improving pipe failure predictions: Factors effecting pipe failure in drinking water networks, Water research (2019) 114926doi:10.1016/j.watres.2019. 114926 . factors, Journal of Hydroinformatics 20 (5) (2018) 1191-1200. doi:10.2166/ hydro.2018.152.

[3] Á. Martínez-Codina, M. Castillo, D. González-Zeas, L. Garrote, Pressure 600 as a predictor of occurrence of pipe breaks in water distribution networks, Urban Water Journal 13 (7) (2016) 676-686. doi:10.1080/1573062X. 2015.1024687.

[4] H. Rezaei, B. Ryan, I. Stoianov, Pipe failure analysis and impact of dynamic hydraulic conditions in water supply networks, Procedia Engineering

119 (1) (2015) 253-262. doi:10.1016/j.proeng.2015.08.883. 
[5] J. Changklom, I. Stoianov, Redundant flow estimation methods for robust hydraulic control in water supply networks, Journal of Hydroinformatics 21 (4) (2019) 571-592. doi:10.2166/hydro.2019.099

[6] N. Fontana, M. Giugni, L. Glielmo, G. Marini, R. Zollo, Real-time control of pressure for leakage reduction in water distribution network: Field experiments, Journal of Water Resources Planning and Management 144 (3) (2018) 04017096. doi:10.1061/(ASCE)WR.1943-5452.0000887

[7] D. Pearson, M. Fantozzi, D. Soares, T. Waldron, Searching for n2: How does pressure reduction reduce burst frequency, in: Proc. IWA Leakage Conf, 2005, p. 13.

[8] A. Kabaasha, J. van Zyl, G. Mahinthakumar, Correcting power leakage equation for improved leakage modeling and detection, Journal of Water Resources Planning and Management 146 (3) (2020) 06020001. doi:10. 1061/(ASCE) WR.1943-5452.0001172.

[9] V. Ghorbanian, B. Karney, Y. Guo, Pressure standards in water distribution systems: reflection on current practice with consideration of some unresolved issues, Journal of Water Resources Planning and Management 142 (8) (2016) 04016023. doi:10.1061/(ASCE)WR.1943-5452.0000665

[10] D. Martínez García, J. Lee, J. Keck, J. Kooy, P. Yang, B. Wilfley, Pressure-based analysis of water main failures in california, Journal of Water Resources Planning and Management 146 (9) (2020) 05020016. doi:10.1061/(ASCE)WR.1943-5452.0001255

[11] Á. Martínez-Codina, L. Cueto-Felgueroso, M. Castillo, L. Garrote, Use of pressure management to reduce the probability of pipe breaks: A bayesian 630 approach, Journal of Water Resources Planning and Management 141 (9) (2015) 04015010.

[12] I. Moslehi, M. Jalili_Ghazizadeh, Pressure-pipe breaks relationship in water 
distribution networks: a statistical analysis, Water Resources Management 34 (9) (2020) 2851-2868. doi:10.1007/s11269-020-02587-4.

[13] D. Wilson, Y. Filion, I. Moore, State-of-the-art review of water pipe failure prediction models and applicability to large-diameter mains, Urban Water Journal 14 (2) (2017) 173-184. doi:10.1080/1573062X.2015.1080848.

[14] A. Scheidegger, J. P. Leitão, L. Scholten, Statistical failure models for water distribution pipes-a review from a unified perspective, Water research 83 (2015) 237-247. doi:10.1016/j.watres.2015.06.027.

[15] A. M. St. Clair, S. Sinha, State-of-the-technology review on water pipe condition, deterioration and failure rate prediction models!, Urban Water Journal 9 (2) (2012) 85-112. doi:10.1080/1573062X.2011.644566.

[16] B. Rajani, Y. Kleiner, Comprehensive review of structural deterioration of water mains: physically based models, Urban water 3 (3) (2001) 151-164. doi:10.1016/S1462-0758(01)00032-2

[17] M. Kutyłowska, Prediction of water conduits failure rate-comparison of support vector machine and neural network, Ecological Chemistry and Engineering. A 23 (2) (2016). doi:10.2428/ecea.2016.23(2)11.

[18] M. Kutyłowska, Forecasting failure rate of water pipes, Water Supply 19 (1) (2019) 264-273. doi:10.2166/ws.2018.078.

[19] Q. Xu, Q. Chen, W. Li, J. Ma, Pipe break prediction based on evolutionary data-driven methods with brief recorded data, Reliability Engineering \& System Safety 96 (8) (2011) 942-948. doi:10.1016/j.ress.2011.03.010.

[20] D. Winkler, M. Haltmeier, M. Kleidorfer, W. Rauch, F. Tscheikner-Gratl, Pipe failure modelling for water distribution networks using boosted decision trees, Structure and Infrastructure Engineering 14 (10) (2018) 14021411. doi:10.1080/15732479.2018.1443145. 
[21] S. Yamijala, S. D. Guikema, K. Brumbelow, Statistical models for the analysis of water distribution system pipe break data, Reliability Engineering \& System Safety 94 (2) (2009) 282-293. doi:10.1016/j.ress.2008.03.011

[22] A. Robles-Velasco, P. Cortés, J. Muñuzuri, L. Onieva, Prediction of pipe failures in water supply networks using logistic regression and support vector classification, Reliability Engineering \& System Safety 196 (2020) 106754. doi:10.1016/j.ress.2019.106754

[23] A. Ng, CS229 - Machine Learning, Lecture Notes: Supervised learning, uRL: https://see.stanford.edu/materials/aimlcs229/ cs229-notes1.pdf. Last visited on 2020/05/11 (2007).

[24] S. A. Andreou, D. H. Marks, R. M. Clark, A new methodology for modelling break failure patterns in deteriorating water distribution sys-

【 tems: Theory, Advances in Water Resources 10 (1) (1987) 2-10. doi: 10.1016/0309-1708(87)90002-9.

[25] Y. Kleiner, B. Rajani, Comprehensive review of structural deterioration of [ water mains: statistical models, Urban water 3 (3) (2001) 131-150. doi: 10.1016/S1462-0758(01)00033-4

[26] J. Røstum, Statistical modelling of pipe failures in water networks (2000). URL http://hdl.handle.net/11250/242082

[27] G. Gangl, D. Fuchs-Hanusch, E. Stadlober, P. Kauch, Analysis of the failure behaviour of drinking water pipelines, Water science and technology: Water Supply 7 (5-6) (2007) 219-225. doi:10.2166/ws.2007.102.

[28] T. M. Inc, stepwiseglm, uRL: https://www.mathworks.com/help/stats/ stepwiseglm.html. Last visited on 2020/05/11 (2020).

[29] R. Silhavy, P. Silhavy, Z. Prokopova, Analysis and selection of a regression model for the use case points method using a stepwise approach, Journal of Systems and Software 125 (2017) 1-14. doi:10.1016/j.jss.2016.11.029 
[30] R. Thomas, S. Peethamparan, Stepwise regression modeling for compressive strength of alkali-activated concrete, Construction and Building Materials 141 (2017) 315-324. doi:10.1016/j.conbuildmat.2017.03.006.

[31] R. Chester, C. Jerosch-Herold, J. Lewis, L. Shepstone, Psychological factors are associated with the outcome of physiotherapy for people with shoulder pain: a multicentre longitudinal cohort study, British journal of sports medicine 52 (4) (2018) 269-275. doi:10.1136/bjsports-2016-096084.

[32] S. I. Vrieze, Model selection and psychological theory: a discussion of the differences between the akaike information criterion (aic) and the bayesian information criterion (bic)., Psychological methods 17 (2) (2012) 228. doi: $10.1037 / \mathrm{a} 0027127$.

[33] J. Kuha, Aic and bic: Comparisons of assumptions and performance, 【 Sociological methods \& research 33 (2) (2004) 188-229. doi:10.1177/ 0049124103262065 .

[34] T. Saito, M. Rehmsmeier, The precision-recall plot is more informative than the roc plot when evaluating binary classifiers on imbalanced datasets, PloS one 10 (3) (2015). doi:10.1371/journal.pone.0118432.

[35] G. Haixiang, L. Yijing, J. Shang, G. Mingyun, H. Yuanyue, G. Bing, Learning from class-imbalanced data: Review of methods and applications, Expert Systems with Applications 73 (2017) 220-239. doi:10.1016/j.eswa. 2016.12 .035

[36] A. Ng, CS229 - Machine Learning, Lecture Notes: Regularization and model selection, uRL: https://see.stanford.edu/materials/ aimlcs229/cs229-notes5.pdf. Last visited on 2020/05/11 (2007). burst rates in water distribution mains, in: Proceedings of the Institution of Civil Engineers-Water Management, Vol. 160, Thomas Telford Ltd, 2007, pp. $73-82$. doi:10.1680/wama.2007.160.2.73. 